Mens

revue d'histoire intellectuelle de l'Amérique française

\title{
Fils légitimes de l'imaginaire national : les Canadiens français selon The Beaver-Canada First, organe des Native Sons of Canada, 1928-1929
}

Sylvie Lacombe

Volume 9, numéro 2, printemps 2009

URI : https://id.erudit.org/iderudit/1023095ar

DOI : https://doi.org/10.7202/1023095ar

Aller au sommaire du numéro

Éditeur(s)

Centre de recherche en civilisation canadienne-française

ISSN

1492-8647 (imprimé)

1927-9299 (numérique)

Découvrir la revue

Citer cet article

Lacombe, S. (2009). Fils légitimes de l'imaginaire national : les Canadiens français selon The Beaver-Canada First, organe des Native Sons of Canada, 1928-1929. Mens, 9(2), 207-241. https://doi.org/10.7202/1023095ar
Résumé de l'article

Cet article décrit la perception des Canadiens français dans le journal Beaver-Canada First, organe officiel de l'association Native Sons of Canada, active dans les années 1920, et qui se présente elle-même comme un groupe de pression destiné à promouvoir un sain patriotisme et une fierté nationale dans tous les champs de la culture et de la société canadienne. L'article décrit l'association et le contenu de son journal avant d'exposer la place ménagée aux Canadiens français; celle-ci est imprégnée de « bonne entente », mais elle témoigne aussi des limites du canadianisme politique, à défaut d'être civique, que professe Native Sons of Canada. 


\title{
FILS LÉGITIMES DE L'IMAGINAIRE NATIONAL : LES CANADIENS FRANÇAIS SELON THE BEAVER-CANADA FIRST, ORGANE DES NATIVE SONS OF CANADA, 1928-19291
}

\author{
Sylvie Lacombe \\ Département de sociologie \\ Université Laval
}

\section{Résumé}

Cet article décrit la perception des Canadiens français dans le journal Beaver-Canada First, organe officiel de l'association Native Sons of Canada, active dans les années 1920, et qui se présente elle-même comme un groupe de pression destiné à promouvoir un sain patriotisme et une fierté nationale dans tous les champs de la culture et de la société canadienne. L'article décrit l'association et le contenu de son journal avant d'exposer la place ménagée aux Canadiens français ; celle-ci est imprégnée de « bonne entente », mais elle témoigne aussi des limites du canadianisme politique, à défaut d'être civique, que professe Native Sons of Canada.

\section{Abstract}

This article describes how French Canadians were depicted in the BeaverCanada First, the official organ of the Native Sons of Canada, an organisation active in the 1920s. The Native Sons presented themselves as a pressure group seeking to promote a bealthy patriotism and national pride in every aspect of Canadian society and culture. The article describes the association and the content of its paper before exploring its portrayal of French Canadians, which was characterized by an attitude of bonne entente and which illustrates the 
limits of the less than civic political 'Canadianism' that the Native Sons of Canada professed.

La décennie des années 1920 apparait à bien des égards comme initiatrice d'une lente transition au cours de laquelle le Canada s'extrait de sa gangue impériale. Progressivement, les relations du Canada avec le monde ne se limiteront plus à l'Empire britannique et bientôt, elles ne passeront même plus par l'Angleterre ${ }^{2}$. C'est cependant avec une grande timidité que le Canada s'adonne aux relations internationales et l'amitié américaine devient rapidement la base étroite de sa politique étrangère ${ }^{3}$. Une transition s'observe aussi sur le plan économique - qui s'achève cette fois plutôt qu'elle ne débute vers un capitalisme industriel qui engendre des divisions régionales et des divisions de classe que les structures politiques existantes n'arrivent pas à transcender. D'où l'émergence de nouveaux partis politiques et mouvements sociaux ${ }^{4}$. La croissance de l'État canadien largement propulsée par sa participation à la Première Guerre mondiale s'accompagne d'un effort d'unification sociale et politique dans une vaste tentative de promouvoir une identité nationale pancanadienne, culminant dans la célébration du jubilé de diamant de la Confédération en $1927^{5}$. En fait, une conscience nationale prend forme à cette période, qui n'est pas un simple produit de l'activité étatique ; elle exsude littéralement des milieux intellectuels, artistiques, religieux - l'Église unie du Canada est créée en 1926 dans une volonté explicite de "canadianiser » les immigrants - et, plus directement lié à notre propos, de la vie associative canadienne ${ }^{6}$. C'est ainsi qu'au début de la décennie 1920, est mise sur pied une association qui accorde une grande valeur au fait d'être né au Canada, Native Sons of Canada (NSC), et qui reprend la devise du mouvement Canada First, ce mouvement apparu au lendemain de la Confé- 
dération, et que Carl Berger identifie comme le précurseur des « impérialistes canadiens ${ }^{7}$ ». Nous reviendrons en conclusion sur cet apparent paradoxe.

Comme plusieurs autres associations nationales formées à la même époque, Native Sons of Canada a contribué à consolider le sentiment national et à promouvoir l'idée d'une distinction canadienne ${ }^{8}$. Notre intérêt pour la place qui y est faite aux Canadiens français tient à deux interrogations. D'abord, comme les Canadiens français peuvent s'enorgueillir d'une longue généalogie en sol canadien, il paraissait logique de se pencher sur la place de cette catégorie de citoyens. Cette place est en partie déterminée par le «bonnententisme » qui caractérise, entre autres, une certaine communauté d'affaires de l'Ontario depuis la Première Guerre mondiale. Le nationalisme professé prétendant être nettoyé de toutes connotations britanniques, la perception des Canadiens français et la place aménagée pour eux dans la Confédération ont ensuite permis d'énoncer des hypothèses sur la contribution canadienne-française dans la construction d'un imaginaire national. Après une présentation relativement détaillée de l'association, de son journal et de ce qu'on y trouve, nous préciserons les occasions où il est question des Canadiens français, pour ensuite proposer une interprétation de leur apport symbolique à l'édification de la nation canadienne. Nous terminons avec un diagnostic global de la vision biculturelle de NSC et de ses limites.

L'association Native Sons of Canada est née à Victoria, Colombie-Britannique, à l'automne 1921 ; elle a reçu sa charte fédérale d'association "patriotique et fraternelle " en février 1922. Son premier Grand Conseil a été formé le 9 mars suivant : les fondateurs visaient à renouer avec la vision des Pères de la Confédération qui leur semblait avoir été perdue?. Inquiets des tensions diverses écartelant alors le pays ${ }^{10}$, ils 
avaient à cœur de créer et promouvoir un esprit national canadien, sans pour autant concevoir ce projet en dehors de l'empire, ni lui donner une couleur antibritannique. Pour arriver à se tailler une place dans le paysage associatif, les membres doivent pourtant dépenser beaucoup d'énergie à souligner leur attachement à l'empire, à la couronne et aux institutions britanniques. En concurrence directe avec les autres associations "fraternelles " du Canada comme Sons of Scotland, Sons of England, ou Sons of Ireland, NSC doit au contraire de celles-ci constamment démontrer qu'elle ne vise pas la destruction de l'Empire britannique. Très proche du nationalisme professé par Henri Bourassa au tournant du $\mathrm{XX}^{\mathrm{e}}$ siècle, le canadianisme de NSC est, comme celui-là l'était déjà, attaqué comme étant antibritannique ${ }^{11}$.

On sait relativement peu de choses des fondateurs et des membres étant donné le caractère secret de cette société qui ne tenait pas de registres sur ses effectifs. Mary Jean Vipond, qui a consacré un chapitre de sa thèse à cette association, avance cependant d'après le nom des membres des conseils exécutifs que la plupart était d'origine britannique, nés en Ontario, mais habitant des villes de l'ouest du pays à la fin des années $1920^{12}$. Si l'on en croit le grand secrétaire, R. D. MacLachlan, ces jeunes hommes - ils étaient trentenaires avaient été membres d'associations locales libérales, conservatrices ou travaillistes; ils étaient de confessions religieuses diverses (presbytérienne, méthodiste, anglicane, catholique) et l'un d'eux était également orangiste ${ }^{13}$. L'association connaît un certain succès : en 1922, il y a 14 assemblées dans la province de la Colombie-Britannique ; en 1924, des assemblées sont constituées en Alberta ; en 1925, une première assemblée ontarienne est formée, qui sera suivie de plusieurs autres, puis une première aussi à Montréal en 1927 - laquelle pour ce qu'on en sait ne sera pas suivie d'autres assemblées 
dans cette province ${ }^{14}$. Dans sa thèse, Vipond estime que 30000 membres est un estimé très modéré de l'effectif de cette association qui avait à la fin des années 1920 plus d'une centaine d'assemblées d'un bout à l'autre du pays, quoique concentrées en Ontario $^{15}$. Ailleurs, cependant, elle parle plutôt de 120000 membres, en précisant toutefois que ce chiffre est non confirmé ${ }^{16}$. À mesure que l'association gagne en assemblées et en membres, son centre de gravité et de décisions se déplace vers l'est pour s'établir à Toronto d'où le journal, The Beaver-Canada First (BCF), sera publié à compter de janvier $1928^{17}$. Mais les membres originaires de la Colombie-Britannique n'en sont pas pour autant dépossédés de leurs rôles de leaders : tout au long de la décennie 1920, les postes de secrétaire, de trésorier et de président national sont comblés par des membres issus de cette province ${ }^{18}$, sauf en 1926 quand C. F. Jamieson, originaire de Lethbridge en Alberta, est élu président $^{19}$. Deux publications mensuelles des NSC ont précédé le $B C F$. La première, Canadian, fut éditée par le Grand Conseil durant quelques mois en 1923 et 1924 ; la seconde, une lettre d'information au titre presque identique, The Canadian, fut publiée par les soins de l'Assemblée $\mathrm{n}^{\circ} 2$ de Vancouver de 1925 à $1927^{20}$. Seul le BCF sera distribué à la grandeur du pays. Il sera remplacé, après avoir fait faillite au début de 1930, par The Native Son qui fera lui aussi faillite en 1934. L'association qui a pu servir de modèle aux fondateurs serait celle des Canadian Club $^{21}$ et, plus vraisemblablement, celle des Native Sons of British Columbia (NSBC) fondée à Victoria en 1899. En effet, sans gommer les différences, importantes, entre NSBC et NSC, l'historien Forrest Pass remarque judicieusement que ces associations ont toutes deux opté pour le modèle d'organisation des fraternités secrètes plutôt que pour celui des rencontres mondaines et élitistes, typiques des Canadian Club. En outre, les deux associations 
partageaient certaines pratiques rituelles et symboliques, notamment lors de l'initiation de leurs membres respectifs. Enfin, il est probable qu'elles aient également tiré leur inspiration première d'organisations américaines en Californie et dans l'État de Washington ${ }^{22}$.

Pour être initié membre des NSC, il fallait être né au Canada, ou être de parents canadiens et avoir résidé au pays avant l'âge de 5 ans; seuil élevé plus tard à 10 ans. Il fallait aussi être "Blanc», une exigence qui trahit l'origine britannocolombienne de l'association. Cette province a en effet été fortement marquée par le racisme antiasiatique durant la première moitié du $\mathrm{XX}^{\mathrm{e}}$ siècle ${ }^{23}$. Une exception était faite vis-àvis des Amérindiens, car en tant que "seniors ", c'est-à-dire descendants d'habitants ayant le plus d'ancienneté au pays, ils faisaient automatiquement partie de l'organisation, pour peu qu'ils en manifestent le désir. À plus d'une reprise, le président revient sur les raisons justifiant ces critères d'admissibilité : les «natifs » canadiens doivent jouer un rôle particulier dans la société, et pour ce faire, avoir une organisation qui leur est propre ${ }^{24}$. C'est à eux qu'échoit la tâche de cimenter le Canada ${ }^{25}$, d'en faire ce que nous appellerions une « totalité nationale ", c'est-à-dire faire en sorte qu'il soit plus que la somme de ses parties - que celles-ci soient des sections religieuses, des factions politiques, des régions géographiques, ou des provinces. Cet aspect est très explicitement formulé dans le mémoire soumis en 1937 à la Commission royale d'enquête sur les relations fédérales-provinciales par NSC : l'intention des Pères de la Confédération, peut-on y lire, étaient de créer un nouvel organisme, plus grand que la somme de ses composantes originelles ${ }^{26}$. L'organisation insiste ainsi sur son caractère non partisan et non confessionnel, et se présente comme un groupe de pression politique dévoué à l'éducation de l'opinion publique, dont la mission est d'apprendre 
aux masses le patriotisme afin de créer une vraie nationalité canadienne. Selon ses propres mots, NSC vise à développer un esprit de tolérance et d'unité, et à promouvoir une fierté nationale dans les arts et professions canadiens. Notons que cette association regroupe moins des membres des élites que "d'honnêtes gens ", pratiquant les petits métiers d'une économie moderne. La plupart des membres sont vendeurs, hommes d'affaires, commis de bureau et instituteurs, mais aux conseils nationaux, on trouve surtout des professionnels, des avocats, des dentistes ${ }^{27}$. Le mouvement est surtout urbain, mais pour ce qui est de la Colombie-Britannique, Pass note tout de même la présence d'assemblées dans des districts ruraux ou des régions d'exploitation forestière ${ }^{28}$.

Parmi les nombreuses causes qui mobilisent NSC, on en dénombre cinq principales qui reviennent systématiquement ; elles touchent pour la plupart la question des symboles nationaux. On veut d'abord un drapeau qui soit distinctement canadien. Jusqu'ici, le Canada a utilisé soit l'Union Jack, que les NSC juge inadéquat parce que non spécifique au Canada, soit le «Red Ensign ", théoriquement réservé à la marine marchande. Ce dernier emblème pourrait éventuellement satisfaire les NSC, mais il faudrait pour cela une prise de position officielle du Parlement ${ }^{29}$. On souhaite aussi remplacer les armoiries par une feuille d'érable. Cet emblème, qu'on juge adapté à la symbolique nationale, remporte l'adhésion d'autres groupes comme les Canadian Club, la Chambre de commerce de l'Ontario, la Conférence des Églises anglicanes, etc. et des publications telles que McLean's, La Presse ou le Manitoba Free Press $^{30}$. On souhaite également la reconnaissance, officielle et explicite, du " $\mathrm{O} \mathrm{Canada}{ }^{31}$ » en tant qu'hymne national et qu'il soit chanté avec le "God Save the King » à la moindre cérémonie. Pour appuyer cette position, on rappelle qu'il a été chanté lors du $60^{\mathrm{e}}$ anniversaire de la Confédération en 1927. 
Selon le Beaver-Canada First, cette version est connue et chantée par tous, à l'exception d'une petite clique de Vancouver. On demande en troisième lieu que les Canadiens de naissance puissent être nommés au poste de gouverneur général du Dominion, et que son titre soit changé pour " vice-roi » puisqu'il ne représente que le roi et non plus le Parlement britannique. En vue de créer une "race » distinctement canadienne, NSC martèle que la nationalité canadienne devrait être reconnue dans les registres de recensement ${ }^{32}$. Conscients que cette création serait toute artificielle, les NSC soulignent que la plupart des Canadiens anglophones ont une origine ethnique mixte et ont en conséquence du mal à n'en choisir qu'une quand vient le temps du recensement. Enfin, dernière demande qui revient comme un leitmotiv, l'abolition des appels au Conseil privé de Londres afin de rendre effective la soi-disant égalité entre tous les membres de l'empire. Dans le mémoire qu'ils soumettent à la Commission royale d'enquête sur les relations fédérales-provinciales, NSC condamne d'ailleurs le Conseil privé pour sa mésinterprétation de la constitution canadienne et de l'esprit de la Confédération qui mine la prééminence de l'État fédéral au profit des juridictions provinciales ${ }^{33}$.

\section{Une célébration de la modernité}

Le Beaver-Canada First $(B C F)$ est un hebdomadaire d'assez grand format qui paraît tous les jeudis de 1928 et de $1929^{34}$, dont le tirage est de 24000 à la fin de la première année et de 34000 un an plus tard. Au début, il est fait d'un seul cahier, puis à compter du numéro 36 , de deux : une section féminine est apparue, la section générale a été incluse dans les pages financières. Dans la section féminine, on trouve des articles sur des femmes qui se démarquent par leur dévouement à une cause, leur patriotisme ou plus simplement par leur sens civique. Elle comprend des extraits d'œuvres de fiction, des 
articles scientifiques (ou pseudo-scientifiques) par exemple sur l'origine des rêves, sur l'évolution de l'architecture domestique, sur l'insanité hier et aujourd'hui, sur l'importance de la vision et de l'usage du sens de la vue dans la vie moderne, ou encore sur les vérités et mensonges de la permanente ${ }^{35}$. On décrit des sites d'intérêt touristique, on reproduit des extraits de conférence, voire des conférences entières, des entrefilets d'autres journaux; etc. Une rubrique d'importance idéologique capitale touche les efforts commémoratifs de l'État fédéral et dans une moindre mesure ceux des gouvernements provinciaux. Dans le travail d'édification de la communauté nationale imaginaire et imaginée ${ }^{36}$ auquel NSC entend contribuer activement, le recours à la "mémoire» des événements passés, des héros ou grands hommes ayant forgé le pays joue, en effet, un rôle clé. Comme ce thème conduit très souvent aux Canadiens français, nous en discuterons plutôt dans la deuxième partie.

Sur le plan idéologique, le journal campe fermement un appel à la réforme du capitalisme. Il défend le libre marché, vante les bienfaits de la concurrence et l'économie capitaliste en général tout en soutenant les projets de pensions de vieillesse, de pensions aux soldats handicapés, aux veuves et orphelins - selon le principe qu'il vaut mieux pécher par générosité que par mesquinerie. Il met en garde contre les solutions faciles. Ainsi, grâce aux machines, on peut fabriquer la même quantité de produits avec moins d'hommes, mais ce faisant, on crée aussi du chômage. Si on augmente au contraire les salaires, on accroît du même coup le pouvoir de consommation de la masse. La première voie conduit à la détresse et trahit la courte vue de l'employeur, qui devient le pire ennemi de son pays, tandis que la seconde voie mène à une prospérité croissante et révèle plutôt un patriote éclairé. Ainsi le bien commun national est l'impératif devant incur- 
ver les réflexes capitalistes ${ }^{37}$. Il affiche aussi une position qu'on qualifiera aisément de féministe : pour le journal, la place des femmes n'est pas à la maison; toutes ne sont pas faites pour la maternité ni pour le mariage. On se moque gentiment des personnages bibliques féminins, qui peuvent difficilement inspirer la femme moderne ${ }^{38}$. Globalement, ce féminisme n'est cependant pas très menaçant pour l'ordre social, d'autant plus que le journal présente aussi la famille (nucléaire) comme le pilier de la vie moderne... tout en soulignant l'inébranlable pertinence de la loi sur le divorce ${ }^{39}$. Mais on affiche aussi des positions plus radicales, demandant par exemple qu'à travail égal, les femmes obtiennent un salaire égal à celui des hommes, condamnant telle femme d'affaires à la tête d'une grande entreprise qui cache son sexe derrière ses initiales, et se satisfait d'une rémunération moins élevée. On exhorte alors les femmes à cesser de se voir elles-mêmes à travers les yeux des hommes $^{40}$.

Une caractéristique frappante du $B C F$ est son engouement pour la modernité matérielle et l'ère du machinisme : des articles qui ne sont pas des publicités s'émerveillent, en le décrivant, $d u$ fonctionnement d'un réfrigérateur ou d'un rasoir électrique ; on se passionne pour la technologie de l'aviation, des automobiles, de la radio et de tout ce qu'on peut faire grâce à ces bienfaits ${ }^{41}$. Cet enthousiasme est à peine tempéré par une mise en garde contre les nouveaux dangers, ou nouvelles nuisances, qui suivent de près les nouveautés technologiques : on avise le lecteur d'être prudent au volant et particulièrement aux passages à niveaux, déplorant le trop grand nombre d'accidents entre voitures et trains; on déplore le vacarme des klaxons qui rendent la ville bruyante ${ }^{42}$.

On s'emballe aussi beaucoup pour les richesses naturelles qui, selon le journal, commencent à peine à être exploitées - plusieurs provinces en seraient encore à prospecter les 
trésors qu'elles recèlent - mais d'ores et déjà, le Canada est une terre d'abondance : premier producteur mondial de nickel, d'amiante, d'hydroélectricité ; il produit le huitième de la production mondiale de blé ; il commence tout juste à produire des plantes textile (lin, chanvre), mais on ne doute pas qu'elles seront bientôt en demande dans le monde entier ${ }^{43}$. On ne compte plus les articles qui glorifient l'une ou l'autre des ressources naturelles canadiennes.

Un autre élément marquant la couleur générale du journal est qu'on y présente la nature comme un immense espace de loisirs (alpinisme, équitation, expédition de canot, de kayak, etc.) où le pays apparaît comme le plus grand terrain de jeux du monde : tous les sports d'hiver et d'été peuvent en effet se pratiquer ici $^{44}$.

Enfin, le sujet récurrent qui traverse tous les numéros du corpus est sans contredit la migration. On critique beaucoup l'immigration britannique sous prétexte que ces nouveaux venus s'installent dans les villes, plutôt que dans les régions de colonisation, et surtout qu'ils obtiennent trop facilement, au détriment des Canadiens de naissance, les emplois de professionnels, de fonctionnaires et de commis de bureau. Ce que demande le journal, c'est de recruter des Canadiens de naissance dans toutes les structures de l'administration civile, municipale, provinciale et nationale, jusqu'à ce que soit obtenue la parité avec le citoyen né en Grande-Bretagne. En outre, une présence trop importante de Britanniques au sein de la population retarde, dit-on, le développement d'un sentiment proprement national car leur affection est tournée vers l'extérieur du pays - l'Angleterre - plutôt qu'en son sein ; la mentalité coloniale s'éternise à cause d'eux.

À l'automne 1928, l'évêque anglican George Exton Lloyd de la Saskatchewan ${ }^{45}$ dénonce sur toutes les tribunes qui s'offrent à lui la soi-disant préférence canadienne pour 
des «bâtards d'Europe de l'est » au détriment d'immigrants britanniques. C'est l'occasion pour le $B C F$ de défendre la diversité ethnique canadienne, en soulignant la sincère loyauté envers leur pays d'adoption chez la plupart des étrangers. On évoque par exemple le courage du mineur slave ou la détermination du Danois, qui franchit les rapides de Lachine en canot, pour conclure qu'une nouvelle civilisation émerge de cette belle diversité, laquelle repose sur l'anglais comme langue commune, le gouvernement démocratique comme système politique, et un système juridique fondé sur les principes britanniques.

Enfin, la question de l'immigration implique invariablement celle de l'émigration aux États-Unis, dont on dit qu'elle est le véritable problème auquel il faut s'attaquer, car les deux questions n'en sont qu'une. Quand on arrivera à garder chez nous nos jeunes adultes parce qu'on pourra leur offrir un travail intéressant et à un salaire compétitif vis-à-vis des salaires américains, alors on n'aura plus besoin d'attirer l'immigrant avec des programmes d'aide économique, il viendra par ses propres moyens tant il sera profitable pour lui de le faire ${ }^{46}$. Comment empêcher nos jeunes travailleurs de partir vers les États-Unis ?.Il faut plus de canadianisme! De la part de nos industriels, cela suppose de mieux payer leur main d'ọuvre ; de la part de nos universités, qu'elles récompensent mieux leurs meilleurs étudiants; et de la part du Canadien moyen, qu'il achète plus de produits canadiens et cesse de consommer des produits européens.

On peut donc caractériser globalement le discours du $B C F$ comme étant résolument modernisateur, et plutôt libéral au sens classique du terme, en sus bien évidemment de son nationalisme prononcé ${ }^{47}$. Voyons donc maintenant quels sont les contextes qui amènent à parler des Canadiens français, et ce qu'on en dit. 


\section{Commémoration et romantisme : nos Anciens}

\section{Canadiens}

NSC salue et soutient les efforts étatiques de construction d'une mémoire nationale en reproduisant, par exemple, le texte de plaques commémoratives inaugurées un peu partout au pays, et en signalant les "lieux de mémoire» et les personnages s'étant illustrés dans le passé ${ }^{48}$. L'association suggère même l'imposition d'un droit d'entrée pour la visite de ces sites afin d'alléger le fardeau de l'État dans son travail de préservation et d'assurer ainsi que tous les édifices historiques dignes d'être conservés le soient ${ }^{49}$. À chaque inauguration de sites ou monuments historiques, à chaque dévoilement de plaques commémoratives, le journal joint avec enthousiasme sa voix à la promotion gouvernementale de l'histoire canadienne. Dans l'ensemble, ces moments sont empreints de romantisme qui exalte le courage, l'héroïsme ou la détermination des personnages du passé. Si elles participent indéniablement d'une idéalisation du passé, ces pages n'en sont pas moins résolument empreintes de modernisme au sens où elles opèrent en même temps un quadrillage culturel du territoire en vue de susciter et d'attirer le tourisme itinérant de masse qui émerge à cette époque $e^{50}$. Là où commémoration et Canadiens français se recoupent, on souligne bien l'enracinement ancestral de ceux-ci en terre nord-américaine et leurs diverses contributions au glorieux passé. On nous décrit, par exemple, le monument élevé en l'honneur de Louis Hébert, premier fermier du pays, près de l'hôtel de ville de Québec, où il figure flanqué de sa femme Marie Rollet à la fière allure ${ }^{51}$. Une plaque au Château Frontenac, puis une autre au parc Montmorency, sont également inaugurées qui commémorent la résistance des Canadiens lors de l'invasion américaine de $1775-1776^{52}$. Chaque fois, les sites sont minutieusement décrits et le texte de l'inscription reproduit dans son 
intégralité. La valorisation de l'histoire canadienne conduit aussi à des articles sur tel ou tel personnage, dont certains sont Canadiens français : il en est ainsi de Madeleine de Verchères, véritable petite héroïne que tout Canadien devrait vénérer ${ }^{53}$, ou de Pierre Lemoyne, sieur d'Iberville, ce meneur d'hommes, ce vaillant soldat, ce brillant diplomate ${ }^{54}$. Le tableau serait incomplet sans les évocations des coureurs des bois, ces véritables pionniers, découvreurs du pays ${ }^{55}$. De même dans le champ de la culture, des artisans ayant fait briller le Canada, parfois jusqu'à l'étranger, grâce à leur art ont droit à des entrefilets, voire à des articles, au moment de leur mort ou peu après. C'est le cas de Louis Jobin, sculpteur et graveur, dont les œuvres sont décrites ${ }^{56}$. On parle aussi d'hommes politiques canadiens-français, contemporains ou d'une autre époque. Une courte biographie de Wilfrid Laurier accompagne l'annonce de l'inauguration de sa maison natale en site historique ${ }^{57}$; elle sera complétée par un plus long article plusieurs mois plus tard dans lequel l'ancien premier ministre est donné comme un modèle de droiture morale ayant, le temps de son passage au pouvoir, triomphé des préjugés religieux et raciaux, car il avait contre lui l'Église catholique de sa province et les orangistes de l'Ontario. Ce qui ne l'a pas empêché de triompher. Le journal admet qu'on peut être en désaccord avec plusieurs de ses politiques, mais qu'on est forcé de reconnaitre la force de sa personnalité. Son charme naturel, l'acuité de son intelligence et le caractère persuasif de ses discours lui ont valu d'être adulé par son propre peuple, mais aussi par les Écossais presbytériens de l'Ontario. Toute sa vie la force de sa nature morale est restée victorieuse contre les tentations opportunistes ${ }^{58}$. Et puis, il y a un portrait flatteur du maire de Montréal, Camilien Houde, dont il faut savoir qu'il est également membre de l'assemblée des Native Sons de Montréal. On le décrit comme le type par excellence d'homme qui s'est fait lui-même, à la droiture irréprochable ${ }^{59}$. 
Dans un autre ordre d'idées, la présence francophone est mentionnée quand on traite de l'aspect multiculturel du pays, soit pour en vanter la tolérance, religieuse ou autre, soit au contraire pour rappeler les limites nécessaires à l'expression de cette tolérance. Tout en soulignant la grandeur de la civilisation et de la culture chinoises, un missionnaire ayant vécu près de vingt ans en Chine propose pourtant une limitation marquée de l'immigration chinoise ${ }^{60}$. Sans qu'on comprenne bien pourquoi, l'auteur paie au passage un tribut à la portion française du Canada pour sa contribution historique ; tout se passe comme si la présence de cet élément français éprouvait déjà suffisamment la tolérance nationale. De même, en évaluant les demandes faites par la communauté ukrainienne de l'Ouest d'enseigner l'ukrainien dans les écoles subventionnées à même les deniers de ses membres, le $B C F$ explique l'impossibilité d'acquiescer à cette demande sans hypothéquer l'avenir du pays : le Canada, répond-il, possède déjà deux langues officielles et, s'il réussit malgré tout à rester efficace et productif en dépit de cette dualité, reconnaittre d'autres langues le conduiraient certainement au désastre. La Chine et l'Inde sont données comme exemples patents de ce danger ${ }^{61}$.

On trouve parfois, plutôt rarement, des extraits de textes en français, un entrefilet reproduit du Devoir affirmant la nécessité d'insuffler une âme canadienne aux immigrants de l'Ouest, des extraits de discours d'Ernest Lapointe, de Camillien Houde ${ }^{62}$, un compte-rendu abrégé d'un discours d'Arthur Sauvééc ${ }^{63}$, chef de l'opposition officielle à l'Assemblée législative de Québec, sur la nécessité de construire le Canada sur le « roc du patriotisme ${ }^{64}$ ». On trouve aussi un résumé de la conférence inaugurale de la $48^{\mathrm{e}}$ réunion annuelle de la Société royale du Canada par $\mathrm{M}^{\mathrm{gr}}$ Camille Roy, recteur de l'Université Laval, dans laquelle son Éminence cite la so- 
ciété scientifique comme étant exemplaire de la bonne entente et de la coopération entre les deux peuples fondateurs au plan intellectuel ${ }^{65}$.

\section{La bonne entente}

De fait, on parle surtout des Canadiens français en se gargarisant de «bonne entente ». Loin d'être une simple posture racoleuse à l'égard des Canadiens français, cette position traduit une sincère volonté de reconnaître l'apport canadienfrançais à l'édification du pays ${ }^{66}$. Ce qui distingue le Canada dans l'Empire britannique, par exemple, est le fait qu'il possède l'unité dans la diversité : les deux « races », de langue, de foi et de mentalité différentes, vivent côte à côte dans la plus parfaite harmonie, et chacune est heureuse de contribuer avec l'autre au bien commun canadien sans pour autant rien sacrifier de son individualité, ni de ses idéaux. Le $B C F$ se garde cependant de nous décrire ceux-ci. Relatant, en s'y associant, les propos d'Henri Bourassa, pour qui la nationalité canadienne repose entièrement sur l'association volontaire et coopérative de tous les groupes du pays, le journal fustige ceux qui voudraient qu'on cesse de tenir compte des Canadiens français dans la gouverne de l'État ${ }^{67}$. Selon nous, le journal déforme sans doute légèrement les propos de Bourassa ici. Quand ce dernier parle des composantes de la nation canadienne, il entend les deux « races » fondatrices (canadienne-française et canadienne-anglaise) et non des groupes, lesquels évoquent moins la dualité canadienne que des immigrants, regroupés selon l'origine ethnique. Les propos de Bourassa sont pourtant connus de NSC. L'association a même parrainé une partie de sa tournée des villes de l'Ouest en 1927 au cours de laquelle il diffusait ce qu'il appelait son « enseignement patriotique $^{68} »$. Par ailleurs, un article de l'organe officiel de l'Assemblée de Vancouver $\mathrm{n}^{\circ} 2$, antérieur à la création de $B C F$, 
présente la conception que se faisait Bourassa de la bonne entente et invite ses lecteurs à épouser la même largeur de vue. Bourassa y précise que l'objet du mouvement ne se restreint pas à un rapprochement entre les provinces du Québec et de l'Ontario, et encore moins se ramène-t-il à la promotion d'une alliance entre les intérêts financiers concentrés à Montréal et à Toronto. La bonne entente vise plutôt à ébaucher une politique véritablement nationale et qui ne serait pas confinée au champ économique étroitement défini. Telle que Bourassa la conçoit, elle comprendrait des mesures pour encourager la colonisation, l'agriculture scientifique et les industries qui en dérivent ; pour faciliter le commerce extérieur ; pour empêcher la concentration excessive de la richesse et du capital dans certaines villes de l'Est ; pour accueillir une immigration intelligemment sélectionnée et faire en sorte qu'elle ne chasse pas nos jeunes vers les villes américaines. Enfin, la bonne entente contribue à l'unité morale et politique du pays en professant un sain canadianisme, au détriment d'un attachement excessif à l'Angleterre et à l'empire ${ }^{69}$.

Gardons en tête cet appel à une vision élargie de la bonne entente pour lire les propos de James Cotton, président de NSC, qui rappelle qu'un des buts explicites de l'association consiste à favoriser la fraternité entre le Québec et l'Ontario, les deux provinces les plus peuplées du pays. Cotton précise que le nombre d'adhérents à NSC s'est même accru à la suite de la publicité de cette mission : renverser les différences de "section » religieuse et de "faction " politique dans un canadianisme exacerbé. On rapporte aussi à cette œuvre l'initiative du premier ministre ontarien qui permet aux enseignants de sa province de suivre des cours d'été au Québec afin de leur apprendre le français ${ }^{70}$. De ce point de vue, on peut avancer que NSC travaille à faire tomber les préjugés de la majorité anglo-canadienne à l'endroit des Canadiens fran- 
çais. Tout comme plusieurs auteurs se réclamant de la bonne entente, NSC avait sincèrement à cœur de reconnaître ce qu'on doit historiquement, culturellement et politiquement aux $\mathrm{Ca}$ nadiens français et de leur aménager en conséquence une place plus étendue dans la vie du Dominion.

À quelques reprises, on traite de l'Assemblée $\mathrm{n}^{\circ} 78$ de Montréal qui, à sa fondation en 1926, comptait dix membres et en comptera cent quarante-sept deux ans après : le président est l'homme d'affaires Gédéon Martel, dont la marque de commerce serait d'être toujours prêt à coopérer pour le bien commun, et le secrétaire, qui occupe également le poste de second vice-président national, J. H. Langevin, lequel peut fièrement faire remonter à plusieurs siècles la venue de ses ancêtres au Nouveau Monde, tous de nobles aventuriers français.

Deux rencontres sont pour NSC l'occasion de se réjouir de ce que ses actions portent fruit : la première a lieu le 5 juillet 1928 et la seconde, le $1^{\text {er }}$ septembre suivant. À la première, une délégation de membres des assemblées de Toronto et d'Ottawa a été reçue par l'Assemblée n ${ }^{\circ} 78$ de Montréal dans un grand banquet. L'accueil et la réception, si l'on en croit le compte-rendu, furent inoubliables; les discours qui y furent prononcés, dont quelques-uns en partie en français, sont ensuite reproduits intégralement dans le journal ${ }^{71}$. On y déclare que les Canadiens français sont admirés et enviés par les autres membres à cause de leur esprit canadien si " naturel et déjà si actif», parce que leur amour du pays natal et du sol inclut tous ceux qui y vivent. Le président Cotton déplore pour sa part le handicap des Canadiens qui ne parlent pas le français, soulignant l'avance des Canadiens français en matière de bilinguisme. Finalement, on célèbre l'unité, celleci étant si nécessaire pour que le Canada devienne une véritable grande nation, et l'on a soin d'ajouter que la dualité lin- 
guistique n'est pas un obstacle à ce rêve - l'exemple de la Suisse servant cette fois à appuyer l'argument. À la seconde rencontre, les assemblées de Toronto rendent la pareille aux assemblées de Montréal et d'Ottawa ${ }^{72}$. Ce soir-là, le président de l'assemblée d'Ottawa ( $\mathrm{n}^{\circ} 86$ ) énonce le souhait que l'entente cordiale entre l'Ontario et le Québec s'enracine profondément dans le cœur des fils canadiens, et vante le Québec comme la province la plus progressiste du Dominion. Il termine son discours en identifiant un vilain défaut que les natifs auraient à corriger : ils connaissent mal leur vaste pays, et sont trop provinciaux dans leurs visions ${ }^{73}$.

Une défense bien sentie des Canadiens français dans les pages du $B C F$ témoigne de la concurrence entre périodiques et entre associations volontaires. Le magazine Saturday Night accuse en effet la presse québécoise de faire campagne contre l'immigration en exigeant que les fonds investis au soutien des immigrants servent plutôt à l'établissement de colons canadiens-français dans les provinces de l'Ouest. Le magazine propose qu'on refuse toute aide économique à quelque colon canadien que ce soit, afin de s'assurer qu'aucun Canadiens français n'en bénéficie. Le $B C F$ s'insurge alors contre l'expression d'une telle mesquinerie, trop heureux de noter que ce sont de semblables attitudes qui empêchent le pays de progresser dans son devenir national ${ }^{74}$. Des loges orangistes, notamment celle de Toronto, font de l'agitation contre NSC qu'elles accusent d'être anti-impérialistes et de défendre le principe du bilinguisme. Le journal revendique comme siennes les deux étiquettes : il est bien anti-impérialiste sans pour autant souhaiter la fin de l'Empire britannique, car il demande simplement qu'on respecte scrupuleusement l'égalité de statut des colonies autonomes et de la Grande-Bretagne au sein du Commonwealth. Et, oui, il défend le bilinguisme au Canada, car selon lui, il est tout naturel de respec- 
ter l'alliance solennelle que le gouvernement de Sa Majesté a nouée avec le peuple français au moment de la conquête britannique ${ }^{75}$.

À la réunion annuelle de la Chambre de commerce de Vancouver, J. H. Woods a prononcé une allocution sur l'esprit de compromis qui fonde l'unité canadienne. Il termine son discours sur une note plus personnelle en avouant être né au Québec et avoir grandi au cœur du Canada français : ces gens, nous dit-il, forment la colonne vertébrale de la vie nationale canadienne; ils vouent une dévotion passionnée au pays. Si l'on veut faire advenir le futur grandiose qui attend le Canada, il faut donc travailler à l'union de sympathie et de compréhension de ses deux grands peuples ${ }^{76}$.

Enfin, un discours du président de King's College à Halifax, le révérend Arthur $\mathrm{H}$. Moore, est reproduit in extenso dans un long article portant sur les charmes du Canada français. L'auteur y avance d'abord que seule la communication entre les deux grandes « races » aux langues et traditions historiques différentes permettra de réaliser leur union, tout comme les moyens modernes de communication ont permis au Canada de vaincre la géographie ${ }^{77}$. Il rappelle ensuite que c'est grâce aux Français que la civilisation est entrée au Canada au XVI ${ }^{e}$ siècle. Le premier trait de caractère du Canadien français est la fierté de sa race, qui le fait vénérer sa mère-patrie : aucun pays plus que la France n'a été ainsi honoré d'un amour si pur. Si l'on avait mieux compris cette caractéristique pendant la Grande Guerre, nous dit le révérend, on aurait sûrement évité la « situation malheureuse » de 1917. Dans une large mesure, le Canada français demeure isolé du reste du Dominion, il n'y souffle pas le vent de l'agitation moderne de la société industrielle, mais plutôt une brise paisible, telle une réminiscence de l'Europe médiévale. Ses habitants chérissent des coutumes et des pratiques d'un autre 
âge qui ne sont pas dénuées de charme, au contraire. Son folklore est tissé de légendes et de superstitions semblables à celles qu'on trouve en Irlande, mais auxquelles s'ajoutent ici des éléments amérindiens ; et le tout forme un amalgame qui rend absolument unique la tradition canadienne-française. Le sens de l'esthétique de ce peuple amoureux de la couleur et de la beauté laisse parfois à désirer, mais il suffit d'écouter chanter les cloches d'un bout à l'autre de la province pour comprendre qu'on y apprécie l'harmonie du son et les arts les plus fins. Le révérend énumère quelques œuvres canadiennes-françaises traduites ${ }^{78}$ et des œuvres anglaises portant sur le Canada français ${ }^{79}$, et précise que l'âme canadienne est une personnalité duale qui ne peut être qu'à demi révélée à ceux qui ne maîtrisent qu'une langue. Si l'on veut atteindre l'unité nationale, on doit reconnaître la nécessité pour les Canadiens de comprendre et l'anglais et le français. On doit apprécier les points de vue respectifs, sympathiser avec les aspirations de l'autre, admirer, respecter les plus hautes et les meilleures traditions, croyances, coutumes qui ont été préservées, et faire advenir une sincère coopération entre les deux "races » dans une atmosphère de confiance et d'estime.

Le ton du révérend Moore est résolument celui de la bonne entente et de la bonne volonté, mais y a-t-il quelque chose de plus substantiel au-delà du romantisme indéniable de cette description ? La question est importante, car à ne s'en tenir qu'à cet aspect romantique, on sera tenté de ramener la place faite aux Canadiens français dans le $B C F$, et plus généralement dans NSC, à celle d'un simple alibi, ou d'une caution, permettant de se doter d'une profondeur historique qui, autrement, ferait défaut. L'engouement pour les Canadiens français serait ainsi à ranger dans le même registre que l'attrait pour les sites historiques et tout ce qui peut être mobilisé pour construire la « mémoire nationale ». On conclurait 
à une forme d'annexion symbolique des Canadiens français du même ordre que celle dont semblent victimes les Amérindiens, ces autres "seniors » que les NSC chérissent. Si l'on s'en tient au $B C F$, l'intérêt pour la réalité et la culture amérindiennes, et pour l'apport amérindien à l'édification de la nation canadienne peut difficilement être qualifié autrement que de vague, de périphérique, voire de simple divertissement. Quand, par exemple, James Cotton nouvellement élu au Grand Conseil, est initié chef des Cris au cours d'une cérémonie à Regina, l'événement est documenté de photos mais très succinctement décrit ${ }^{80}$, et surtout, n'est pas l'occasion d'une réflexion, même sommaire, sur la vie dans les réserves, sur les dépossessions territoriales qu'impliquait la marche vers l'Ouest au lendemain de la Confédération, ni sur l'iniquité du statut d'Amérindien. Tout au plus le $B C F$ mentionne-t-il parmi les progrès qu'il reste encore à accomplir, la nécessité d'instruire les Amérindiens et déplore-t-il que les "Esquimaux » meurent de faim sur la banquise quand ceux d'Alaska et du Groenland prospèrent ${ }^{81}$.

De même, le portrait global qu'on dresse du Canada français, et des Canadiens français, en est un largement convenu à l'époque, exaltant la vie communautaire. Les Canadiens français semblent être invoqués pour s'émouvoir d'un passé qui se meurt partout ailleurs ; ils sont touchants comme le sont de précieux artéfacts d'une époque révolue. Mais cette image d'un passé traditionnel idéalisé est aussi celle que le Québec donne de lui-même dans ses publicités en Ontario et aux États-Unis à la même époque, à travers par exemple les ouvrages de sa Commission des monuments historiques ${ }^{82}$. Elle n'est donc pas propre à NSC et illustre plutôt à quel point le journal de l'association est marqué par le contexte général de l'époque. Jusqu'à présent, on a vu que la perception des $\mathrm{Ca}$ nadiens français dans le $B C F$ s'inscrit dans une vision plus 
large, «bonnententiste », qui renvoie par moments à une conception biculturelle de la nation canadienne mais qui s'accommode aussi des clichés passéistes et communautaristes sur le Canada français.

Nous allons maintenant aborder des articles où les $\mathrm{Ca}$ nadiens français sont moins les respectueux descendants des aieux de la nation qu'une avant-garde tournée vers l'avenir, et le faisant même advenir. Cette autre image concerne les relations internationales du Canada. Le thème par excellence pour l'association est, bien sûr, le canadianisme sous toutes ses formes. Or, pour ce qui est de la promotion de l'entité canadienne sur le plan international, au sein de l'empire et face aux autres nations, les Canadiens français sont présentés comme ayant une solide longueur d'avance sur leurs compatriotes anglophones parce qu'ils sont spontanément enclins à voir le Canada comme une entité politique se suffisant à ellemême ${ }^{83}$ et parce que leur amour du pays atteint le statut de véritable culte; vénérant leur terre natale, leur amour inclut en outre tous ceux qui sont nés sur son sol ${ }^{84}$. Un premier article, incluant une petite portion mal écrite en français, porte sur un jeune homme, Roche [sic] Pinard ${ }^{85}$ du collège SainteMarie de Joliette, ayant remporté le concours canadien d'éloquence avec une allocution portant sur le « Canada parmi les nations du monde "; le discours est prononcé dans sa langue maternelle, et néanmoins acclamé tant par le jury que par l'audience en dépit du fait que plusieurs parmi celle-ci, précise le journal, ne comprennent pas le français! Le jeune Pinard traite du Canada en tant que nation libre ayant conquis, par sa probité, le droit à la vie internationale. Il expose la situation duale du pays en insistant sur les avantages qu'elle procure. En bref, deux génies remarquables s'y trouvent, le génie latin et le génie saxon : le premier est littéraire et philosophique tandis que le second est commercial et pragmati- 
que. Se félicitant au passage du fait que la rivalité entre les deux « races » soit en voie de s'éteindre, Pinard célèbre le fait que les Canadiens d'aujourd'hui parlent les deux langues dominantes dans le monde, l'anglais étant la langue des affaires et le français celle de la diplomatie, toutes deux nécessaires pour une vie internationale riche et élaborée. La rencontre, nous dit le journal, s'est terminée dans l'enthousiasme et sur l'air d'O Canadd ${ }^{86}$. Un second article reproduit la traduction anglaise d'un discours prononcé par Ernest Lapointe au Cercle universitaire de Montréal en octobre 1927, intitulé «Le statut international du Canada » dans lequel le ministre canadien de la Justice retrace la participation canadienne à divers événements ou conflits internationaux et insiste surtout sur l'importance de la déclaration Balfour au terme de la Conférence impériale l'année précédente ${ }^{87}$. Finalement, on trouve un condensé anglais d'une série de conférences prononcées par Rodolphe Lemieux à la Sorbonne sur «l'évolution politique du Canada » qui décrivent le long chemin parcouru par le Canada de son statut de colonie à celui de nation, détaillant les diverses étapes de l'extension progressive son autonomie politique $^{88}$.

\section{$* * *$}

Selon Vipond, le «nativisme » des NSC est sans aucun doute l'élément le plus important du nationalisme qu'ils professent, et il ne s'agit pas ici de remettre en question le lien qu'elle établit entre leurs intérêts économiques, de classes moyennes, et leurs positions idéologiques ${ }^{89}$. Ils convoitaient certainement les postes de commis de bureau, d'employés des grands magasins, de fonctionnaires, d'administrateurs, etc., que les immigrants britanniques obtenaient facilement et au détriment des Canadiens de naissance. Mais on aurait tort de réduire leur nationalisme à cette seule réalité. À l'instar d'autres 
organisations et associations des années 1920 qui ont contribué au développement d'un sentiment national, NSC a instigué un changement de perceptions en martelant de plusieurs façons, et pour différents domaines de la vie collective, la nécessité pour les Canadiens de se doter d'un imaginaire proprement national, de ne pas se satisfaire du formidable développement économique depuis le début du $\mathrm{XX}^{e}$ siècle, mais de travailler aussi à développer une communauté d'esprit, "d'âme », en chérissant les premiers symboles distinctifs canadiens. Prises globalement, ces expressions d'une conscience nationale naissante transcendent les classes sociales, les allégeances politiques et les différentes origines ethniques ${ }^{90}$.

Bien que NSC se réclame explicitement du mouvement «Canada First », né juste après la Confédération, l'association n'en altère pas moins le sens de la devise en se l'appropriant. Car le sentiment national des fondateurs de «Canada First » est si largement coloré d'appartenance impériale que l'objectif qu'ils cherchent à atteindre est une restructuration importante de l'Empire britannique, non l'émancipation politique du Canada du giron impérial ${ }^{91}$. Dans les décennies qui ont suivi, l'attachement à l'appartenance canadienne a pu progresser ainsi au sein de la matrice impériale, sans générer de contradictions insurmontables chez les Canadiens d'origine britannique. Même au plus fort de l'ambition impériale canadienne-anglaise ${ }^{22}$, celle-ci comprend et s'appuie sur un fort sentiment nationaliste ${ }^{93}$. Mais la situation a été toute différente pour les Canadiens français qui, sans nécessairement rejeter le lien à la Couronne britannique, avaient beaucoup moins d'affinité avec son empire, et saisissaient déjà le besoin de symboles nationaux.

Sans souhaiter rompre explicitement avec l'Empire britannique, NSC exprime néanmoins un nationalisme totalement émancipé de l'ambition impériale. Pour eux, le Canada 
n'est pas une " totalité partielle » de l'empire ; son particularisme et son unité ne lui viennent pas de son insertion dans l'ensemble impérial. Il constitue au contraire une entité autonome, qui se suffit à elle-même. Le $B C F$ reconnaît précisément aux Canadiens français une importante contribution dans le développement d'un tel esprit proprement national, et dans l'évolution historique du pays en tant qu'entité autonome au sein de l'Empire britannique. On aura remarqué une importante similitude de vues entre cette position et le canadianisme professé par Henri Bourassa depuis le tournant du $\mathrm{XX}^{\mathrm{e}}$ siècle. Celui-ci insiste beaucoup, en effet, sur l'absence de communauté d'intérêt a priori entre la Grande-Bretagne et le Canada, cherchant à soustraire le pays de l'influence impériale et travaillant à développer la prise en compte des intérêts strictement canadiens auprès des hommes politiques. Il fait reposer sur les épaules des Canadiens français la responsabilité de propager le patriotisme canadien qu'ils ressentent si spontanément. Ce devoir, moral et politique, implique l'égalité de droit absolue entre les deux groupes sociolinguistiques dans toute l'étendue du pays ${ }^{94}$. C'est ici que la communauté de vues entre Bourassa et NSC s'affaiblit car l'égalité entre les Canadiens, français et anglais, et ce qu'elle implique, n'est défendue que du bout des lèvres dans les pages du $B C F$. Celui-ci n'aborde en tous cas jamais la question épineuse des droits des Canadiens français dans les provinces anglophones et se contente, en somme, de vœux pieux à l'égard du biculturalisme. Si NSC partage bien une bonne part de l'ambition nationale de Bourassa, cela concerne donc moins le biculturalisme cher au politicien que son interprétation des relations étrangères du Canada, notamment avec la Grande-Bretagne, et son anticipation de l'indépendance inévitable de la colonie. 
Cette réserve faite, on peut néanmoins avancer que NSC témoigne du fait que la transition du Canada impérial au Canada-entité nationale s'est solidement appuyée sur les Canadiens français, et sur leur attachement national. Les aspirations nationales de ceux-ci, leur nationalisme, a donc eu un impact directement positif dans la constitution, au moins imaginaire, du Canada. En minimisant le caractère britannique, dans son acception ethnique, de la nation canadienne, NSC a formulé l'idée d'une citoyenneté politique dont le critère d'appartenance est le fait d'être né sur son territoire. Le pancanadianisme de l'association est théoriquement fondé sur la notion d'un melting pot dont les éléments disparates communient dans le même désir de faire du pays une grande nation indépendante.

Se voulant inclusif, ce nationalisme présente pourtant deux restrictions majeures : d'abord le brassage ethnique ne concernait que les immigrants "blancs » et les Amérindiens, et il excluait les Noirs ainsi que certains groupes d'origine asiatique. Sur ce plan, NSC n'a jamais réussi à se départir de son racisme grossier. La seconde restriction, plus importante pour notre propos, concerne la valorisation des Canadiens français dont il faut bien voir qu'elle ne remet pas en question la notion de "réserve française » confinée au territoire du Québec. Si le $B C F$ excite l'admiration de ses lecteurs pour les $\mathrm{Ca}-$ nadiens français, s'il les enjoint à imiter ceux-ci, il ne soutient jamais la nécessité de leur reconnaitre des droits dạns les provinces autres que le Québec. Leur pancanadianisme s'en trouve limité et peine à projeter dans l'avenir les implications de la vision ébauchée. 


\section{NOTES}

${ }^{1} \mathrm{Je}$ remercie les évaluateurs de Mens dont les judicieux commentaires ont permis de bonifier la présente version.

${ }^{2}$ En 1923 est conclu un traité entre le Canada et les États-Unis qui, pour la première fois, est signé sans l'intervention d'un représentant du gouvernement britannique. L'année suivante, 1926, un représentant canadien permanent est, pour la première fois, en poste à Washington. Il s'agit de Vincent Massey. William Lewis Morton, The Canadian Identity, Madison, University of Wisconsin Press, 1961, p. 71.

${ }^{3}$ John Herd Thompson et Allen Seager, Canada 1922-1939: Decades of Discord, Toronto, McClelland and Stewart, 1985, $438 \mathrm{p}$.

${ }^{4}$ William Lewis Morton, The Progressive Party in Canada. Toronto, University of Toronto Press, 1950, $331 \mathrm{p}$.

${ }^{5}$ Robert Cupido, «Appropriating the Past: Pageants, Politics, and the Diamond Jubilee of Confederation ", Revue de la Société bistorique du Canada, vol. 9, n”1 (1998), p. 155-186.

"Mary Jean Vipond, National Consciousness in English-Speaking Canada in the 1920': Seven Studies, Thèse de doctorat (philosophie), University of Toronto, 1974,558 p.

${ }^{7}$ Carl Berger, The Sense of Power: Studies in the Ideas of Canadian Imperialism, 1867-1914, Toronto, University of Toronto Press, 1970, p. 49-77.

${ }^{8}$ Margaret E. Prang, « Nationalism in Canada's First century », Journal of the Canadian Historical Association, 1968, p. 114-125.

"J. H. L. Morgan, "Native Sons of Canada. Its Origin and Accomplishments", Beaver-Canada First (BCF), vol. I, no17 (17 mai 1928), p. 4.

1" On sait que durant les années qui précèdent le déclenchement de la Grande Guerre, et pendant celle-ci, les relations entre Canadiens français et Canadiens anglais sont pour le moins tendues. Voir entre autres Carl Berger, Imperialism and Nationalism, 1884-1914. A Conflict in Canadian Thought, Toronto, Copp Clark Publishing, 1969, 119 p. et Sylvie Lacombe, La rencontre de deux peuples élus. Comparaison des ambitions nationale et impériale au Canada, 1896-1920, Québec, Presses de l'Université Laval, 2002, 291 p. Mais les années 1920 sont également celles du mécontentement des Maritimes et des fermiers de l'Ouest. Voir à ce titre Thompson et Seager, Canada 1922-1939.

${ }^{11}$ Voir Lacombe, La rencontre de deux peuples élus. 
${ }^{12}$ Ibid. p. 271.

${ }^{13}$ R. D. MacLachlan, "Charges Against the NSC », $B C F$, vol. II, n" 10 (21 février 1929), p. 7.

${ }^{14}$ Bien que Vipond (National Consciousness in English-Speaking Canada in the 1920 's, p. 271, note 9) avance qu'il y avait plusieurs assemblées canadiennesfrançaises en 1929, nous n'avons pas trouvé mention d'autres assemblées au Québec que celle de Montréal ( $\left.\mathrm{n}^{\circ} 78\right)$ dans les numéros du Beaver-Canada First, l'organe officiel des NSC, qui se trouvent à Bibliothèque et Archives Canada à Ottawa. Nous avons épluché la totalité de ces 84 numéros qui vont du vol. I, $\mathrm{n}^{\circ} 17$ (17 mai 1928) au vol. III, $\mathrm{n}^{\circ} 2$ (26 décembre 1929) (30 numéros dans le volume I ; 52 numéros dans le volume II et 2 numéros dans le volume III). Nous n'avons pas non plus trouvé d'archives ni aucun type de documents produits par l'assemblée $\mathrm{n}^{\circ} 78$ de Montréal. Cela aurait éventuellement permis de mettre en relief ou de relativiser, en tous cas d'approfondir, notre étude de la perception des Canadiens français par cette association.

${ }^{15}$ Ibid., p. 268.

${ }^{16}$ Mary Jean Vipond, «Nationalism and Nativism: The Native Sons of Canada in the 1920s ", Canadian Review of Studies in Nationalism, vol. IX, n" 1 (1982), p. 83.

${ }^{17}$ Pour faire l'étude présentée ici, nous avons traité le journal comme un auteur unique sans distinguer les articles signés des autres (non signés), beaucoup trop nombreux, et sans non plus chercher à dégager une quelconque transformation dans le temps, étant donné la trop courte période couverte par le corpus (avril 1928 à décembre 1929).

${ }^{18}$ Forrest Duncan Pass, Pacific Dominion: British Columbia and the Making of Canadian Nationalism, 1858-1958, Thèse de doctorat (histoire), University of Western Ontario, 2008, p. 221.

${ }^{19} \mathrm{~J}$. H. L. Morgan, « NSC, Its Origins and Accomplishments », BCF, vol. I, $\mathrm{n}^{\circ}$ 21 (14 juin 1928), p. 16.

20 Pass, Pacific Dominion, p. 221, note 77.

${ }^{21}$ Vipond, National Consciousness in English-Speaking Canada in the 1920's, p. 269.

${ }^{22}$ Pass, Pacific Dominion, p. 226 et suiv.

${ }^{23}$ Notons cependant que la constitution de NSBC, qui était globalement plus à droite que NSC, ne comptait aucune exclusion raciale avant 1925 quand les 
critères d'admissibilité ont été modifiés pour exclure les personnes d'ascendance asiatique. Voir Forrest Duncan Pass, « The Wondrous Story and Traditions of the Country: The Native Sons of British Columbia and the Role of Myth in the Formation of an Urban Middle Class », BC Studies, $\mathrm{n}^{\circ} 151$ (automne 2006), p. 16.

${ }^{24}$ Voir, par exemple, «Nebulous Body », BCF, vol. I, nº 31 (23 août 1928), p. 3 et « Why Native Sons Only ? », BCF, vol. I, n” 35 (27 septembre 1928), p. 7.

${ }^{25}$ Voir, par exemple, L. S. McGill, « Pellets of Canadianism », (BCF, vol. II, $\mathrm{n}^{\circ}$ 8 [7 février 1929], p. 16) où l'auteur, président de l'assemblée Smithers, décrit le peuple canadien comme un mur de maçonnerie qu'on est en train de construire avec des pierres de différentes formes et tailles ; les natifs sont le mortier qui peut faire tenir ce disparate ensemble.

${ }^{26}$ J. B. Coyne, A. R. M. Lower, R. O. MacFarlane, Brief submitted to Royal Commission on Dominion-Provincial Relations by Native Sons of Canada, Winnipeg, Albion Press, 1937, p. 6.

${ }^{27}$ Vipond, National Consciousness in English-Speaking Canada in the 1920's, p. 270-272.

${ }^{28}$ Pass, Pacific Dominion, 2008, p. 24.

${ }^{29}$ Les premières discussions « sérieuses » à propos du drapeau canadien ne prendront place à la Chambre des Communes qu'après la Deuxième Guerre mondiale. Voir à ce sujet José E. Igartua, The Other Quiet Revolution. National Identities in English Canada, 1945-1971, Vancouver, UBC Press, 2006, 277 p.

30) Vipond, National Consciousness in English-Speaking Canada in the 1920's, p. 282.

${ }^{31}$ Le juge en chef R. S. Weir avait écrit à l'occasion du $300^{\circ}$ anniversaire de la ville de Québec en 1908 une version anglaise de la chanson de Basile Routhier sur l'air composé par Calixa Lavallée. C'est cette version, au détriment de celle de T. B. Richardson, qui deviendra la norme nationale (H. V. Nelles, The Art of Nation-Building. Pageantry and Spectacle at Quebec's Tercentenary, Toronto, University of Toronto Press, 1999, p. 239). Le O Canada n'a cependant détrôné le God Save the King que beaucoup plus tardivement dans le XX' siècle. La plupart du temps les deux chants étaient interprétés lors des cérémonies publiques.

${ }^{32}$ Cette catégorie n'apparaîtra dans les formulaires de recensement qu'en 1996.

${ }^{33}$ Coyne, Lower et MacFarlane, Brief Submitted to Royal Commission..., p. 22-25. 
${ }^{34}$ La collection de Bibliothèque et Archives Canada s'arrête au vol. III, $\mathrm{n}^{\circ}$ 2, daté du 26 décembre 1929. Nous n'avons pas trouvé d'autres exemplaires conservés dans d'autres centres d'archives ou bibliothèques. Selon Vipond pourtant, la publication s'arrête au début de l'année 1930. Vipond, National Consciousness in English-Speaking Canada in the 1920's, p. 278, note 23.

${ }^{35}$ Sur le sens civique féminin, voir les mots sur Mme J. L. Callan, première femme à occuper un poste de commissaire scolaire dans une petite ville ontarienne $\left(B C F\right.$, vol. I, $\mathrm{n}^{\circ} 36$ [4 octobre 1928], p. 16) et sur la très populaire juge Emily Murphy, engagée dans une croisade contre la drogue $(B C F$, vol. I, $n^{\circ} 42$ [15 novembre 1928], p. 9) ; sur l'évolution de l'architecture, $B C F$, vol. II, nos 7, 8 et 9 (31 janvier, 7 février et 14 février 1929); sur les déséquilibres moraux, $B C F$, vol. II, n" 6 (24 janvier 1929), p. 10 ; sur la vue, voir l'article de l'opticien, C.H. Tuck, $B C F$, vol. II, no 19 (25 avril 1929), p. 11 ; sur le soin des cheveux, « Hair Facts and Fallacies », BCF, vol. II, n" 1 (20 décembre 1928), p. 5 .

${ }^{36}$ L'expression est à prendre au sens où l'emploie Benedict Anderson, c'està-dire non en tant que cette communauté serait fictive, mais plus simplement en tant qu'elle relève de représentations sociales grâce auxquelles une société s'interprète elle-même et s'institue dans le même mouvement. Benedict Anderson, Imagined Communities. Reflections on the Origin and Spread of Nationalism. Londres, Verso, 1983, $160 \mathrm{p}$.

${ }^{37}$ «Unfair Importation of Labor », BCF, vol. II, n" 7 (31 janvier 1929), p. 1. Voir aussi « Accessory Before the Fact », BCF, vol. II, n" 9 (14 février 1929), p.16.

${ }^{38}$ "Marriage Vows and Business... Which ? », BCF, vol. I, n" 39 (25 octobre 1928), p. 9 ; et «Are Reformers A Menace ? », BCF, vol. II, n" 1 (20 décembre 1928), p. 16.

${ }^{39}$ "The Divorce Question », BCF, Vol II, n 8 (7 février 1929), p. 16.

4" "Do Women in Business Always Get a Square Deal ? », BCF, vol. II, n" 3 (3 janvier 1929), p. 10.

${ }^{41}$ Grâce à la voiture, la nature devient accessible aux citadins, par exemple ; la radio permettra à des hommes sans qualités de diffuser leur message au plus grand nombre et la manipulation des foules par les grands orateurs ne sera plus possible ; la radiodiffusion rendra en outre la culture accessible à tous, etc. 
42 "The Price of Speed», BCF, vol. II, n" 22 (16 mai 1929), p. 16 ; «Just Noise ", BCF, vol. II, n" 13 (14 mars 1929), p. 16.

${ }^{43}$ Le joumal aime à démonter le "préjugé » voulant que les exportations canadiennes vers les États-Unis soient des matières premières et que les importations en provenance des États-Unis soient des produits manufacturés à partir de ces dernières.

${ }^{44}$ Plusieurs articles portent sur l'un ou l'autre sport, mais pour avoir une bonne idée de la posture générale du journal, voir " The World's Playground ", $B C F$, vol. I, n" 41 (8 novembre 1928), p. 14 ; et « Winter Recreation ", $B C F$, vol. II, n 5 (17 janvier 1929), p. 14.

${ }^{45}$ Plusieurs références véhémentes sont faites à l'évêque anglican : "An Unjustified Complaint » (BCF, vol. I, $\mathrm{n}^{\circ} 26$ [19 juillet 1928], p. 4), « Keeping the British Out » (BCF, vol. I, $\mathrm{n}^{\circ} 38$ [18 octobre 1928], p. 16), «Immigration Propaganda » (BCF, vol. I, n 46 [13 décembre 1928], p. 4). Dans ce dernier article, on soupçonne l'évêque d'être l'auteur d'une série de lettres anonymes envoyées dans les journaux de l'Ouest, lettres dont le ton revanchard est empreint de rancœur à l'égard des Canadiens d'origine étrangère, et demandant comme une litanie toujours plus d'immigrants britanniques. Le prolifique évêque a aussi fondé la National Association of Canada afin de mieux coordonner les efforts pour contrer l'immigration européenne continentale qu'on désignait euphémiquement comme étant en provenance de pays « non préférés ». Voir Howard Palmer, Patterns of Prejudice. A History of Nativism in Alberta, Toronto, McClelland and Stewart, 1982, p. 98 et suiv.

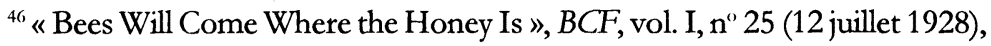
p. 3.

${ }^{47}$ Sur ces deux points, NSC se distingue nettement de NSBC, laquelle était beaucoup plus critique à l'égard de la modernité et de ses effets sur la vie communautaire et sociale. Pass évoque l'insistance de NSBC sur le passé provincial antérieur à la Confédération dans l'élaboration des figures inspiratrices proposées à ses membres, et bien sûr, elle donnait corps à une identité régionale, et non nationale. Voir Pass, Pacific Dominion, p. 34 et suiv.

${ }^{48}$ " Preserving and Restoring Our Historic Sites », BCF, vol. I, $\mathrm{n}^{\circ} 27$ (26 juillet 1928), p. 2.

49 "Preservation of the Old », BCF, vol. II, n" 2 (27 décembre 1928), p. 16.

${ }^{50}$ "Travel, Why, How, Where ?», BCF, vol. I, n 37 (11 octobre 1928), p. 1 ;B. P. McCafferty, "Historic Spot Recalls the Past », BCF, vol. I, n" 23 (28 juin 
1928), p. 6 ; et « More than 10 Million Visit Ontario from States », $B C F$, vol. I, n" 31 (23 août 1928), p. 5.

${ }^{51}$ "Louis Hebert », BCF, vol. I, n" 25 (12 juillet 1928), p. 12.

${ }^{52}$ "Commemorate Defenders of Quebec », BCF, vol. I, n" 31 (30 août 1928). À noter que le numéro précédent du 23 août 1928 est également numéroté 31. Le même article sera réimprimé tel quel dans un numéro subséquent (vol. I, n" 33 [13 septembre 1928], p. 2).

${ }^{53}$ " Madelaine de Vercheres », BCF, vol. 2, no 14 (21 mars 1929), p. 8.

${ }^{54} B C F$, vol. II, no 16 (4 avril 1929), p. 4.

${ }^{55}$ "Canada's Romantic History », BCF, vol. I, n" 46 (13 décembre 1928), p. 9.

${ }^{56} \mathrm{La}$ Sainte Vierge sur l'escarpement du Saguenay, ainsi que des autels et des chapelles dans des églises de Beaumont, de Lévis et de la Visitation. « Jobin, the Wood Carver ", BCF, vol. I, n" 26 (19 juillet 1928), p. 3.

${ }^{57} B C F$, vol. I, no 24 (5 juillet 1928), p. 2.

${ }^{58}$ " Laurier ", BCF, vol. II, n" 11 (28 février 1929), p. 16.

${ }^{59}$ "Who is Houde ?», BCF, vol. I, n" 33 (13 septembre 1928), p.1.

${ }^{61}$ " Rev. A. W. Lochead Addresses Assembly NSC on the Oriental Question ", BCF, vol: I, n" 19 (31 mai 1928), p. 7.

${ }^{61}$ " Ukrainianism Vs Canadianism », BCF, vol. I, n" 26 (19 juillet 1928), p. 8.

${ }^{62}$ "Quebec Political Leader Discusses Canadian Mind », BCF, vol. II, $\mathrm{n}^{\circ} 39$ (12 septembre 1929), p. 3.

${ }^{63}$ Qu'on orthographie « Saure ».

${ }^{64}$ A. Saure, "Canada Must be Grounded on the Bedrock of Patriotism ", $B C F$, vol. I, n 38 (18 octobre 1928), p. 3.

${ }^{65}$ « Maintain Two Great Cultures », BCF, vol. II, n²6 (20 juin 1929), p. 1.

${ }^{66}$ Une étude intéressante du mouvement de la bonne entente remet en question le diagnostic classique de l'historiographie québécoise qui ramène ce mouvement à un simple subterfuge pour faire accepter la conscription aux Canadiens français : voir Robert Talbot, « Une réconciliation insaisissable : le mouvement de la bonne entente, 1916-1930», dans Mens, vol. VIII, n" 1 (automne 2007), p. 67-125. Notre propre analyse du $B C F$ va dans le même sens que le propos de Talbot. 
${ }^{67}$ "Where Mr Bourassa Stands », BCF, vol. I, n" 28 (2 août 1928), p. 9.

${ }^{68}$ Robert Rumilly, Henri Bourassa : la vie publique d'un grand Canadien, Montréal, Chanteclerc, 1953, p. 701 ; Pass, Pacific Dominion, p. 223.

69" Bonne Entente », dans The Canadian, vol. I, n” 4 (décembre 1925/janvier 1926), p. 2-3. Je remercie Forrest D. Pass, qui m'a gracieusement fourni une copie de cette coupure.

${ }^{70}$ « Banquet Held by Grand Council in Hotel Saskatchewan ", BCF, vol. I, $\mathrm{n}^{\circ}$ 30 (16 août 1928), p. 1.

${ }^{71}$ " Quebec and Ontario Meet in the Hearts of the NSC », BCF, vol. I, $\mathrm{n}^{\circ} 25$ (12 juillet 1928), p. 7. En réponse à un toast invitańt les congressistes à se fondre dans un véritable canadianisme, l'assistance s'est spontanément mise à chanter un " $\mathrm{O}$ Canada » si vibrant que l'édifice en aurait été ébranlé !

${ }^{72}$ «Toronto District Council Banquet Visitors », BCF, vol. I, n 33 (13 septembre 1928), p. 7.

${ }^{73}$ W. C. McCarthy, « Canada Our Native Land », BCF, vol. I, n" 26 (19 juillet 1928), p. 2.

${ }^{74}$ «A French Canadian Plot », BCF, vol. I, n’ 38 (18 octobre 1928), p.16.

${ }^{75}$ «The Hidden Hand of Rome », BCF, vol. I, n" 40 (1 $1^{\text {cr }}$ novembre 1928), p. 1. Notons que c'est plutôt à l'égard de l'Acte de Québec en 1774 qu'on peut évoquer une alliance avec les colons français leur permettant de parler leur langue et de pratiquer leur religion et leurs lois civiles sous régime anglais. Voir aussi R. D. MacLachlan, «Charges Against Native Sons of Canada », $B C F$, vol. II, n"10 (21 février 1929), p. 7.

${ }^{76} \mathrm{~J}$. H. Woods, "Spirit of Compromise the Formula for Canadian Unity ", $B C F$, vol. II, n" 17 (11 avril 1929), p. 3.

${ }^{77}$ A. H. Moore, «The Charm of French Canada », BCF, vol. II, n" 28 (27 juin 1929), p.1.

${ }^{78}$ The Canadians of Old de Philippe Aubert de Gaspé, traduit par Charles G. D. Roberts ; les petites pièces du juge Rivard ; et, bien que son auteur soit français, Maria Chapelaine de Louis Hémon, traduit par M. Blake.

${ }^{79}$ Plusieurs volumes de poèmes par W. H. Drummond ; C. W. Colby, Canadian Types of Old Regime; Frank Oliver Call, The Spell of French Canada.

${ }^{80}$ À l'Exposition de Régina, des bandes amérindiennes étaient présentes, et le chef de l'une d'elles, Red Dog, a souhaité instituer le président de NSC grand 
chef des Cris ; la cérémonie s'est déroulée en présence de M. Graham, de la Commission indienne, et de $\mathrm{M}$. Christianson, inspecteur des réserves. « Banquet Held by Grand Council in Hotel Saskatchewan », BCF, vol. I, n 30 (16 août 1928), p.1.

${ }^{81}$ «Educating Our Indians Is an Important Task », BCF, vol. II, n" 39 (12 septembre 1929), p. 3. Selon Pass aussi, les sympathies vis-à-vis des peuples amérindiens sont empreintes de romantisme et nourrissent l'idée d'une « indianité authentique », Pacific Dominion, p. 232.

${ }^{82}$ Voir Pierre-Olivier Ouellet, « Nos routes se couvrent de touristes à la recherche de nos reliques du passé. Les débuts de la Commission des monuments historiques (1922-1928) ", dans Revue d'bistoire de l'Amérique française, vol. 61, $\mathrm{n}^{\circ} 2$ (2007), p. 235-251.

${ }^{83}$ "Toronto District Council Banquet Visitors », BCF, Vol I, n" 33 (13 septembre 1928), p. 7.

${ }^{84}$ « Banquet Held by Grand Coucil in Hotel Saskatchewan », BCF, vol. I, n 30 (16 août 1928), p. 1.

${ }^{85}$ Il s'agit sans doute de Roch Pinard.

${ }^{86}$ "French Canadian Youth Captures Imagination of Toronto Audience ", $B C F$, vol. II, $\mathrm{n}^{\circ} 25$ (6 juin 1929), p. 7.

${ }^{87} B C F$, vol. I, no 30 (16 août 1928), p. 5. Elle a été traduite par Alexandre Clément.

${ }^{88}$ "Political Evolution of Canada », BCF, vol. II, n" 3 ( 3 janvier 1929), p. 15 ; idem, vol. II, $\mathrm{n}^{\circ} 4$ (10 janvier 1929), p. 4 ; idem, vol. II, $\mathrm{n}^{\circ} 5$ (17 janvier 1929), p. 15 ; idem, vol. II, n" 6 (24 janvier 1929), p. 3.

${ }^{89}$ Vipond, "Nationalism and Nativism ». Pass insiste également sur une identité de classe, essentiellement de classe moyenne, pour comprendre le rôle important joué tant par NSBC que NSC en Colombie-Britannique : voir "The Wondrous Story and Traditions of the Country " et Pacific Dominion.

${ }^{90}$ Prang, « Nationalism in Canada's First century ».

${ }^{91}$ Berger, The Sense of Power, p. 49-77.

${ }^{92}$ C'est-à-dire pendant la Guerre sud-africaine, dite des Boers (1899-1902) et pendant la Première Guerre mondiale.

${ }^{93}$ Lacombe, La rencontre de deux peuples élus.

${ }^{94}$ Ibid., chap. 2. 